\title{
Producción más limpia y el manejo de efluentes en plantas de harina y aceite de pescado
}

\section{RESUMEN}

La aplicación de estrategias ambientales como la Producción Más limpia dentro de las operaciones del sector pesquero, específicamente plantas productoras de harina y aceite de pescado, ha permitido generar una reducción significativa de la contaminación al medio ambiente marino, a través de la recuperación de compuestos valiosos presentes principalmente en los efluentes que eran retornados al mar y que ahora son introducidos en el proceso principal, mejorando de este modo no solo el desempeño ambiental sino también aumentando la productividad y contribuyendo positivamente sobre la imagen de la organización ante los diversos grupos de interés.

Palabras clave: desempeño ambiental, efluentes, grupo de interés, plantas de harina y aceite de pescado, producción más limpia

Cleaner Production AND efFluent MANAGEMENT IN FLOUR PLANTS AND FISH OIL

\section{ABSTRACT}

The implementation of environmental strategies such as Cleaner Production in operations, specifically plants producing fishmeal and fish oil, fisheries sector has generated a significant reduction of pollution to the marine environment through the recovery of valuable compounds present mainly in effluents were returned to the sea and are now introduced in the main process, thereby improving not only environmental performance but also increasing productivity contributing positively on the image of the organization to the stakeholders.

Keywords: cleaner production, effluent, environmental performance, fishmeal industry stakeholders

\section{INTRODUCCIÓN}

El Perú es el mayor productor y exportador de harina y aceite de pescado a nivel mundial y de la misma forma, la pesquería industrial se constituye en la mayor fuente contaminante del medio ambiente marino - costero, en la cual se destaca el vertimiento de efluentes de desembarque de anchoveta y de la producción industrial de harina de pesado (agua de cola, sanguaza y agua de bombeo). Esto se ve claramente reflejado pues, en los últimos 5 años las bahías de El Ferrol, Chimbote y Paita, muestran un alto nivel de contaminación producto de la actividad reductora de la materia orgánica presente en los efluentes. Pero a pesar que algunas de plantas del sector cuentan con emisores submarinos, los contaminantes se siguen vertiendo al mar a mayor o menor distancia de la costa [10]. De aquí la necesidad de que estos efluentes reciban un tratamiento previo a su vertimiento al mar.

Además, al ser el medio marino la fuente para la producción el este sector, surge la necesidad de lograr la sostenibilidad ${ }^{1}$ de los recursos pesqueros manteniendo la salud del ecosistema a través de las regulaciones dadas por el estado al sector pesquero industrial, las cuales se ven potenciadas por iniciativas privadas mediante la aplicación de estrategias ambientales como la Producción más limpia, que además de traer beneficios directos sobre el medio ambiente, mejoran el desempeño ambiental en el sector y contribuye positivamente sobre la imagen de la organización ante los diversos grupos de interés.

\section{MARCO TEÓRICO}

\section{Producción Más Limpia}

Uno de los medios por los cuales se busca proteger el medio ambiente es la Producción Más Limpia (PML) por la cual se busca la eliminación o reducción de las materias primas tóxicas, reducción de emisiones, vertimientos y desechos y el uso eficiente de los recursos. Es decir, la PML además de pensar en "qué hacer con los residuos", piensa en "qué hacer para no generarlos".

Ingeniero Agroindustrial de la Universidad Nacional de Trujillo. E-mail: perla_paredes@hotmail.com

1 La sostenibilidad implica la reconversión tecnológica para volver los procesos más eficientes en el uso de materiales, insumos y recursos naturales. Esta reconversión implica el uso de tecnologías como parte de un sistema de producción limpia que minimicen los desperdicios y diseños ambientales de producto que permitan su reproceso. 


\section{Definición}

El término Producción más Limpia fue introducido por la Oficina de Industria y Medio Ambiente del Programa de las Naciones Unidas para el Medio Ambiente (PNUMA) en 1989, como la "aplicación continua de una estrategia ambiental preventiva integrada a procesos productivos y servicios para mejorar la ecoeficiencia ${ }^{2}$ y reducir los riesgos humanos y al medio ambiente".

Lo que se busca con el proceso de Producción Más Limpia es evitar una generación excesiva de residuos, dado que por un lado es considerada una pérdida económica como producto del mal aprovechamiento de los recursos e insumos empleados, y por el otro, los residuos son contaminantes y afectan a la salud y al ambiente, por lo que su reducción permite prevenir impactos ambientales negativos [4].

En este sentido, la producción más limpia tiene como objetivo principal lograr la reducción de los impactos ambientales del proceso productivo, enfocándose en la mejora de procesos y productos con el fin de evitar problemas ambientales antes de que ocurra [3], [6], [2].

$\mathrm{Si}$ se compara Producción Más Limpia y Ecoeficiencia se tiene que este último es un concepto más abarcativo que incluye no solo la prevención de la contaminación ${ }^{3}$ (Pollution Prevention), sino también la reducción en el origen, la reducción de la intensidad de materias primas, el uso racional de la energía, y una serie de prácticas y búsquedas sistemáticas de alternativas, "Mejora Continua" y de métodos de producción más limpia, evitando y superando las practicas end-of- pipe, o al final del caño, modificando y dándo un nuevo enfoque a los procesos, es decir "reinventando" procesos.

Dentro de las prácticas de Producción Más Limpia se encuentran las buenas prácticas operativas, la substitución de insumos, el mejor control de los procesos, la modificación del equipo, cambio de

2 Es un término relacionado a la producción más limpia, utilizado por primera vez en la Cumbre de Río de Janeiro en 1992, que se define como la producción de bienes y servicios con menor consumo de insumos y materias primas y menor contaminación ambiental. El concepto de la ecoeficiencia usado en el Perú implica producir o aprovechar los recursos, añadir valor a los productos y servicios, consumiendo menos materias primas, generando menos contaminación o deterioro ambiental a través de procedimientos ambiental y económicamente eficientes. Este concepto está estrechamente ligado con el desarrollo sostenible, pues busca la optimización de dimensiones de desarrollo en forma paralela: el crecimiento económico, la equidad social y el valor ecológico.

3 Prevención de la contaminación es el uso de procesos, prácticas y/o productos que permiten reducir o eliminar la generación de contaminantes en sus fuentes de origen; es decir, que reducen o eliminan las sustancias contaminantes que podrían penetrar en cualquier corriente de residuos o emitirse al ambiente (incluyendo fugas), antes de ser tratadas o eliminadas, protegiendo los recursos naturales a través de la conservación o del incremento en la eficiencia. tecnología, reutilización ${ }^{4}$, recuperación y reciclaje in situ, producción de subproductos útiles y la reformulación y rediseño del producto [7].

\section{Beneficios}

Se puede resumir los beneficios de la producción más limpia en que permite la optimalización del proceso y ahorro de costos mediante la reducción y el uso eficiente de materias primas e insumos en general, el mejoramiento de la eficiencia operativa de la planta, el mejoramiento de la calidad de los productos y consistencia porque la operación de la planta es controlada y por ende más predecible; permite la recuperación de algunos materiales de los subproductos, la reducción de los residuos, y por ende, reducción de los costos asociados a su correcta disposición, permite también minimizar emisiones y/o descargas en la fuente, reduciendo riesgos para la salud humana y ambiental y elevando simultáneamente la competitividad [1] y finalmente permite el mejoramiento de la imagen de la empresa ante clientes, proveedores, socios, comunidad, entidades financieras, etc. [13].

\section{Producción más limpia como estrategia empresarial}

En la actualidad, la producción más limpia no es vista por la mayoría de empresas del sector industrial como una estrategia viable económicamente, por tal motivo estas empresas optan por la adopción de estrategias ambientales correctivas denominadas tratamientos al final de proceso (o al final de tubo). Sin embargo, comparando los cambios que se generan en la estructura de costos totales, cuando se decide invertir en producción más limpia y cuando no, se tiene que con el tiempo los costos disminuyen significativamente, debido a los beneficios generados a partir del aumento en la eficiencia de los procesos, los ahorros en el consumo de materias primas y energía, y la disminución de residuos y emisiones contaminantes.

Todo este ahorro de la estructura de costos totales sin producción limpia versus aquella con producción limpia y aún más con tecnología limpia, hace que la producción más limpia sea vista como una estrategia empresarial orientada hacia procesos productivos, productos y servicios, para fortalecer la competitividad empresarial mediante innovaciones tecnológicas, reducción de costos, y disminución de riesgos en aspectos de seguridad, salud humana y medio ambiente. La esencia de esta estrategia de carácter preventivo, busca el uso

4 Es decir, la reutilización de materiales residuales dentro del mismo proceso para otra aplicación en beneficio de la empresa. 
eficiente de energía, agua e insumos, así como el aprovechamiento de residuos, integrando al mismo tiempo beneficios económicos, ambientales y sociales.

Se considera que las estrategias de producción más limpia inicialmente solo requieren un cambio en las prácticas culturales o pequeños cambios instrumentales (BPM, BPA, entre otras) hasta que llega un momento en el que se agotan estas opciones, haciéndose necesario invertir en nuevas tecnologías conocidas como "tecnologías limpias". Las tecnologías limpias contribuyen a la mejora de la eficiencia del proceso en cuanto que disminuye el tiempo de operación, haciendo un menor y mejor uso de materia prima, insumos y servicio; aunque requiere de un elevado costo de inversión [13].

Crecientemente, los países y las empresas que son más competitivos no son aquellos que acceden a los más bajos costos de los recursos, sino aquellos que emplean las tecnologías y los métodos más avanzados para utilizar esos recursos.

Paulatinamente más países están interesados en la implementación de tecnologías limpias y prácticas sostenibles con el ambiente, debido a que la conciencia sobre la problemática ambiental ha crecido en los últimos años; aunque el proceso es lento, cada vez más la empresas adoptan procesos y tecnologías eficientes, sujetos a producción más limpia, lo que les contribuye, no solo al cumplimiento de la normatividad, sino a las exigencias de acceso a diferentes mercados y al aumento de la productividad y competitividad. Por ello la PML es una estrategia eficaz y eficiente para reducir los impactos ambientales de una empresa, pues promueve la sustentabilidad de los negocios [13].

\subsection{Manejo de efluentes y $P M L$}

Actualmente, la implementación de medidas de producción más limpia viene a ser el primer paso que debe realizarse a la hora de manejar los efluentes en una empresa.

La ventaja de aplicar prácticas de PML está en que promueve el uso eficiente de materias primas, agua y energía, entre otros insumos, a fin de eliminar o reducir en las fuentes de origen la cantidad de residuos no deseados que se genera durante los procesos de producción. De esta manera, además de reducir los costos unitarios de producción, se reducen los requerimientos para el tratamiento final de desechos, si éste fuera necesario, y, por ende, se reduce el costo de adquisición de una planta de tratamiento y de sus consecuentes costos de operación y mantenimiento.
Por lo tanto, para disminuir costos de producción, es necesario reducir los flujos de residuos; o que, para reducir los flujos de residuos, es necesario incrementar la eficiencia productiva, lo que también lleva a disminuir los costos de producción. Una conclusión lógica de esta afirmación, es que la opción de introducir prácticas de PML debe ser considerada como prioritaria y en forma exhaustiva antes de abordar soluciones de tratamiento "al final del proceso".

Las técnicas de PML pueden aplicarse a cualquier proceso industrial, y abarcan desde cambios operacionales relativamente fáciles de ejecutar hasta cambios más profundos, como la sustitución de insumos, la modificación de procesos $u$ operaciones unitarias, o el uso de tecnologías más limpias y eficientes [3].

\section{Enfoque Piramidal en el manejo de efluentes}

Las técnicas del manejo de residuos o, mejor dicho, del manejo de efluentes (han ido evolucionando. Esquemáticamente, la Figura 1 ilustra lo que se ha denominado como "Enfoque piramidal para el manejo de efluentes", el cual consiste en agotar las soluciones basadas en prácticas de producción más limpia, antes de intentar el manejo de flujos de residuos como desechos al final del proceso de producción. Este último consiste en realizar el tratamiento y la disposición final de residuos considerados como desechos.

Dentro de las prácticas de PML, se debe priorizar aquellas de prevención de la contaminación y de eficiencia energética frente a las prácticas de reciclaje, reuso y recuperación.

Existen ciertos flujos de residuos generados cuya cantidad no puede ser reducida, o no es fácil de reducir, mediante prácticas de prevención de la contaminación o de eficiencia energética; como la sangre de un matadero de reses; las plumas de un matadero de pollos; el pelo o sus formas proteicas degradadas del proceso de pelambre en una curtiembre; el agua de refrigeración que sale caliente; el calor sensible del vapor ya condensado, entre muchos otros flujos de residuos similares. En estos casos, se debe proceder directamente con las prácticas de reciclaje, reuso y/o recuperación. Sin embargo, no se debe perder de vista que las operaciones asociadas a estas prácticas (reciclaje, reuso y/o recuperación) pueden hacerse de manera más eficiente mediante prácticas de Prevención de la Contaminación (PC) y de Eficiencia Energética $(E E)$. Es decir que, estas últimas (PC y EE), deben 
Figura 1. Enfoque piramidal para el manejo de efluentes.

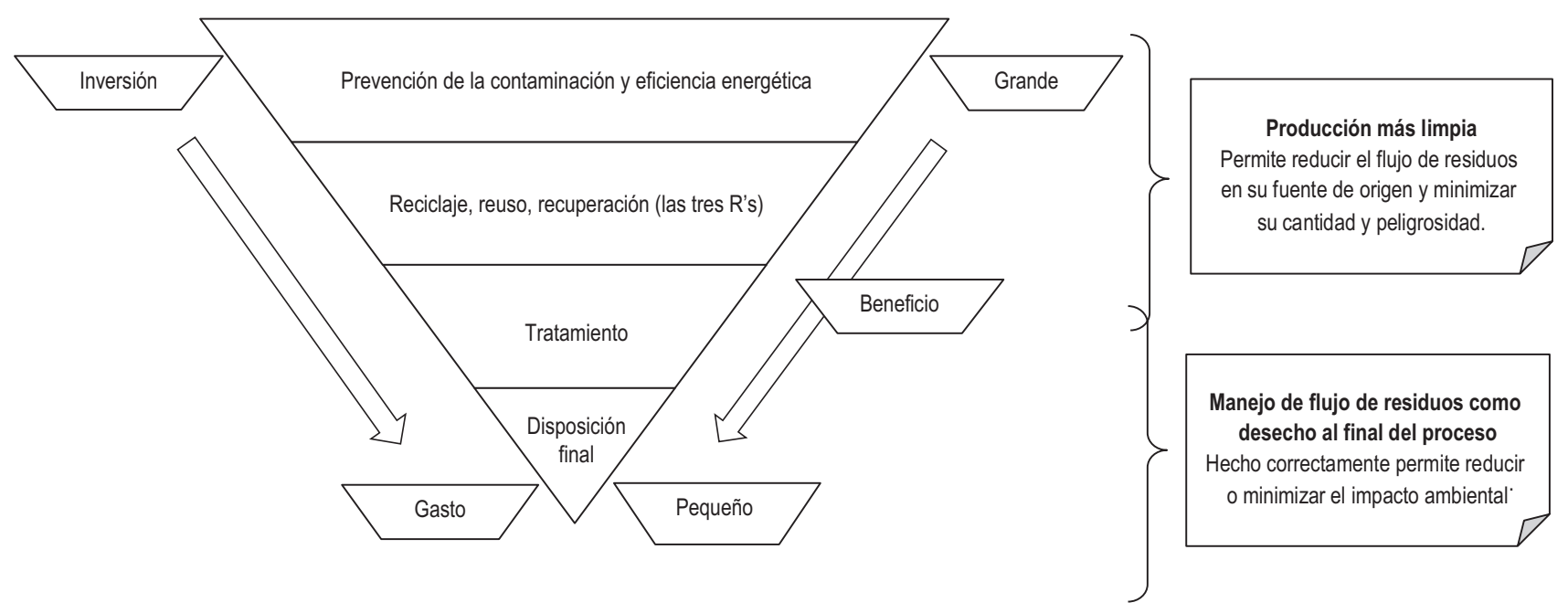

Fuente: Guía Técnica General de Producción Más Limpia, 2005.

ser aplicadas a los procesos empleados en el reciclaje, reuso y/o recuperación.

En general, los recursos empleados para introducir prácticas de PML en una empresa son considerados como una inversión, normalmente de corto plazo, ya que generan retornos económicos y beneficios ambientales simultáneamente. Contrariamente a ello, los recursos empleados para hacer el manejo de residuos como desechos al final del proceso productivo (plantas de tratamiento) son considerados como un gasto, ya que no generan retornos económicos, excepto por el beneficio que resulta por evitar que se generen impactos ambientales, beneficio que para la empresa tiene un carácter intangible en la mayoría de los casos [3].

\subsection{Producción Más Limpia para la recuperación de sólidos de efluentes de plantas de harina y aceite de pescado}

El Ministerio del Ambiente declara la necesidad de promover la inversión privada en procesos productivos que utilicen tecnologías e insumos limpios y el desarrollo de procesos de reconversión de las industrias contaminantes. Al efecto, se establece una doble estrategia. La primera se basa en la consolidación de los mecanismos de comando y control (leyes, normas y fiscalización). Y la segunda, se sustenta en la promoción de medidas voluntarias estimuladas por el desarrollo del mercado.

\section{Mecanismos de control gubernamental}

Considerando que las principales formas de contaminación de las plantas de harina y aceite de pescado provienen de las emisiones gaseosas, residuos sólidos y efluentes, y puesto que el control de este último ha cobrado mayor relevancia en los últimos años, en la Tabla 1 se presentan los Límites Máximos Permisibles (LMP) para los efluentes de la industria pesquera de Consumo Humano Indirecto (CHI) que fueron aprobados en virtud de la Ley 28817, a través del Decreto Supremo 010-2008-PRODUCE. Esta norma busca que se incremente la protección de la calidad ambiental marino-costera con el tiempo, y por ello se ven 3 categorías de cumplimiento [11]. 
Tabla 1. Límites Máximos Permisibles para los efluentes de la industria pesquera

\begin{tabular}{|c|c|c|c|c|c|}
\hline & I & II & III & & \\
\hline $\begin{array}{l}\text { Parámetros } \\
\text { Contaminantes }\end{array}$ & $\begin{array}{l}\text { Límites } \\
\text { máximos } \\
\text { permisibles de } \\
\text { los efluentes } \\
\text { que serán } \\
\text { vertidos dentro } \\
\text { de la zona } \\
\text { de protección } \\
\text { ambiental litoral } \\
\text { (a) }\end{array}$ & $\begin{array}{l}\text { Límites } \\
\text { máximos } \\
\text { permisibles de } \\
\text { los efluentes } \\
\text { que serán } \\
\text { vertidos fuera } \\
\text { de la zona } \\
\text { de protección } \\
\text { ambiental } \\
\text { litoral (a) }\end{array}$ & $\begin{array}{l}\text { Límites } \\
\text { máximos } \\
\text { permisibles de } \\
\text { los efluentes } \\
\text { que serán } \\
\text { vertidos fuera } \\
\text { de la zona } \\
\text { de protección } \\
\text { ambiental } \\
\text { litoral (b) }\end{array}$ & Método de análisis & Formato \\
\hline $\begin{array}{l}\text { Aceites y } \\
\text { Grasas (A y } G \text { ) }\end{array}$ & $20 \mathrm{mg} / \mathrm{l}$ & $1,5 * 103 \mathrm{mg} / \mathrm{l}$ & $0.35^{*} 103 \mathrm{mg} / \mathrm{L}$ & $\begin{array}{l}\text { Standard Methods for } \\
\text { Examination of Water and } \\
\text { Wastewater, 20th. Ed. Method } \\
5520 \text { D. Washington; o Equipo } \\
\text { Automático Extractor Soxhlet. }\end{array}$ & $\begin{array}{l}\text { Los valores } \\
\text { consisten en } \\
\text { el promedio }\end{array}$ \\
\hline $\begin{array}{l}\text { Sólidos } \\
\text { suspendidos } \\
\text { Totales (SST) }\end{array}$ & $100 \mathrm{mg} / \mathrm{l}$ & $2,5^{*} 103 \mathrm{mg} / \mathrm{l}$ & $0.70 * 103 \mathrm{mg} / \mathrm{L}$ & $\begin{array}{l}\text { Standard Methods for } \\
\text { Examination of Water and } \\
\text { Wastewater, 20th. Ed. } \\
\text { Part.2540D Washington }\end{array}$ & $\begin{array}{l}\text { diario de un } \\
\text { mínimo de } \\
\text { tres muestras } \\
\text { de un }\end{array}$ \\
\hline $\mathrm{pH}$ & $6-9$ & $5-9$ & $5-9$ & $\begin{array}{l}\text { Protocolo de Monitoreo } \\
\text { aprobado por Resolución } \\
\text { Ministerial Nº 003-2002-PE. }\end{array}$ & $\begin{array}{l}\text { según se } \\
\text { establece en } \\
\text { la Resolución }\end{array}$ \\
\hline $\begin{array}{l}\text { Demanda } \\
\text { Bioquímica } \\
\text { de Oxígeno } \\
\text { (DBO5) }\end{array}$ & $<60 \mathrm{mg} / \mathrm{l}$ & (c) & (c) & $\begin{array}{l}\text { Resolución Ministerial Nº } 003- \\
\text { 2002-PE (d). }\end{array}$ & $\begin{array}{l}\text { Ministerial No } \\
\text { 003-2002-PE }\end{array}$ \\
\hline
\end{tabular}

(a) La Zona de Protección Ambiental Litoral establecida en la presente norma es para uso pesquero.

(b) De obligatorio cumplimiento a partir de los dos (2) años posteriores a la fecha en que sean exigibles los LMP señalados en la columna anterior.

(c) Ver Segunda Disposición Complementaria y Transitoria.

(d) El Protocolo de Monitoreo será actualizado.

Fuente: Decreto Supremo 010-2008-PRODUCE.

Los LMP establecidos en este reglamento, son de cumplimiento obligatorio para los establecimientos industriales pesqueros o plantas de procesamiento nuevos y para aquellos que se reubiquen. De esta forma, ningún establecimiento industrial pesquero o planta de procesamiento podrá operar si no cumple con los LMP señalados en la Tabla 1.

Para cumplir los LMP establecidos, los titulares de los establecimientos industriales pesqueros deberán implementar sistemas de tratamiento químico, bioquímico u otros complementarios al tratamiento físico.

\section{Medidas voluntarias, aplicación de tecnologías limpias}

El sector pesquero industrial en el Perú ha aplicado paulatinamente la estrategia de PML a través del empleo de tecnología limpia. Es así que las principales empresas pesqueras productoras de harina y aceite de pescado cuentan desde el 2000 con un sistema primario de recuperación de sólidos compuesto en la mayoría de casos por: un separador de sólidos, una trampa de grasa y una celda de flotación por aire disuelto. Así mismo se realizaron cambios drásticos en los sistemas de descarga de materia prima con la introducción de equipo de traslado al vacío utilizando bombas $y$ sistemas de tuberías de plástico. Esta inversión en nuevas tecnologías limpias como el cambio de los equipos de proceso de secado, así como las modificaciones a los equipos existentes han contribuido significativamente con la eficiencia del proceso y la reducción de las descargas 
principalmente de emisiones líquidas y gaseosas al medio ambiente.

La implementación de una nueva etapa para recuperación de sólidos del agua de bombeo mejora los estándares de calidad y eficiencia en la elaboración de harina de pescado. La legislación concerniente se centra en la recuperación de sólidos y grasas, por lo que se espera que la inversión en tecnología ambiental redundara en una mejor productividad.

Si bien la mayoría de plantas ya cuenta con los sistemas de tratamiento exigidos, la eficiencia de los procesos de tratamiento de las aguas de bombeo es todavía muy baja. Después de tratados y antes de ser vertidos al mar, los efluentes aun presentan niveles muy altos de aceite y grasas, sólidos suspendidos y ejercen altos niveles de demanda biológica o (bioquímica) de oxígeno. Además, muchas de las plantas sobre todo en la zona norte del litoral, no cuentan con emisores $y$ vierten sus efluentes directamente frente a las plantas. Siendo el impacto directo de estos vertimientos, la mortalidad masiva de la biota $^{5}$ marina. Ante esta situación, la Dirección General de Asuntos Ambientales de Pesquería (DIGAAP) viene desarrollando importantes actividades para eliminar y mitigar los efluentes de la actividad pesquera mediante la exigencia de los programas de adecuación y manejo ambiental (PAMA)y de los estudios de impacto Ambiental (EIA) y últimamente con la exigencia de la actualización de los mismos, planes de manejo ambiental (PMA), para adecuar las actividades a los límites máximos permisibles (LMP) de la actividad pesquera, en el marco de la política nacional ambiental del Ministerio del Ambiente (MINAM) [10].

La historia de la pesquería peruana ha sido una batalla continua por alcanzar una producción sostenible, capaz de generar beneficios continuos para la población actual sin limitar las capacidades productivas de las generaciones futuras, ni comprometer la integridad del mar peruano.

La contaminación ambiental es algo que todos tememos que estar involucrados porque afecta de manera directa, con repercusiones en nuestra salud y a la salud ambiental. En la pesquería industrial, los efluentes generados del desembarque de las bolicheras y los vertimientos de las plantas harineras presentan elevados residuos orgánicos que resultan en la mortalidad masiva de los recursos de la pesca artesanal y la acuicultura, generando

5 Flora y Fauna. pérdidas masivas de millones de dólares y serios impactos sociales.

\section{Caso de éxito}

Las empresas pesqueras han empezado a optar por tecnología de producción más limpia que permitan reducir el impacto contaminante al medio ambiente a la vez que recuperan material valioso que es incorporado en el proceso productivo principal.

La empresa pesquera Tecnológica de Alimentos S.A (TASA) en su planta de Pisco, en el 2004 inició el proyecto para la implementación de una sistema de recuperación de sólidos y aceites presentes en el agua de bombeo ${ }^{6}(A B)$ que de ser vertida al mar sin previo tratamiento, generaría problemas de contaminación marina, atentando contra las actividades de pescadores artesanales, contra las poblaciones de fauna marina y el equilibrio ecológico en general.

Así también los otros efluentes del proceso, como el agua de cola y la sanguaza, que antiguamente eran descargados al mar, ahora son retornados al proceso de elaboración de harina y aceite de pescado.

Esta inversión en tecnologías de tratamiento del $A B$ realizada por TASA, trae consigo, además de los beneficios ambientales, una mayor rentabilidad económica gracias a la recuperación de estos elementos y su reincorporación al proceso de elaboración de harina y aceite de pescado.

El sistema básico de tratamiento consiste inicialmente en la separación de los sólidos del componente líquido del $\mathrm{AB}$, mediante un tamiz rotativo filtrante o Trommel. La masa sólida es retornada a la línea de proceso mientras que el agua y aceite son enviados a una trampa de grasas para recuperar el aceite flotante, que es luego conducido al proceso de elaboración de aceite (de recuperación o aceite PAMA $^{7}$ ) que representa un ingreso considerable para la empresa. El agua con sólidos en suspensión es dirigida a una segunda fase de tratamiento. Esta consiste en una celda de flotación que produce una espuma que es recuperada con una espumadera rotativa, que luego es llevada al proceso de aceite PAMA. Finalmente, el agua remanente es conducida a una tercera fase de coagulación, floculación y flotación por aire disuelto o DAF químico, donde se genera un volumen de lodo húmedo que es compactado finalmente con una separación en frío (Figura 2)

6 Agua de mar que forma parte del fluido mediante el cual el pescado es bombeado a la planta harinera.

7 Aceite de pescado que ha sido obtenido del agua de bombeo a través del proceso de recuperación mediante sistemas de flotación por aire disuelto. 
logrando reducir la humedad del lodo hasta un $70 \%$. El efluente líquido del DAF en este punto ha sido ya clarificado y cumple con los estándares internacionales de calidad de descargas líquidas de fábricas pesqueras. Todo el sistema está diseñado con líneas de retorno para tener finalmente una sola línea de efluente al mar.
Los lodos que son obtenidos de la separadora ambiental pueden son deshidratados y convertidos luego en harina de pescado de calidad estándar, que a su vez puede ser homogenizada con harinas de diferente calidad, o bien utilizada como insumo en la elaboración de piensos y alimento balanceado para ganado y piscifactorías [5].

Figura 2. Sistema de recuperación de grasa y sólidos del agua de bombeo plantas de harina y aceite de pescado.

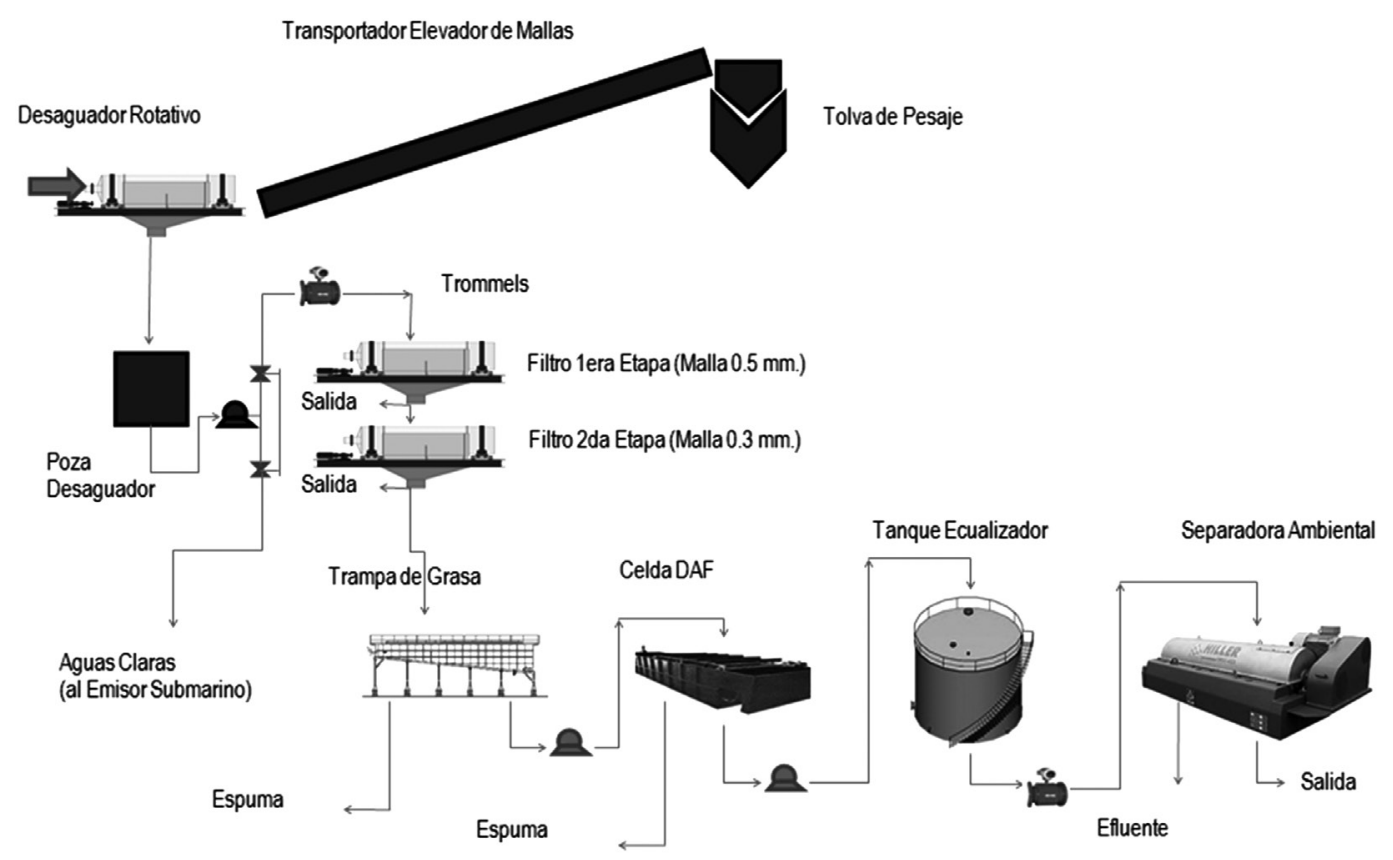

El resultado de la implementación de esta serie de equipos y tecnologías de tratamiento representa una recuperación de $95 \%$ de los sólidos y grasas presentes en el $A B$, los cuales hasta hace pocos años eran descargados directamente en el mar, hoy están siendo recuperados mediante procesos fisicoquímicos de coagulación, floculación y deshidratación [5].

Se ha estimado que la recuperación representa un incremento del $4 \%$ de la producción total de harina de pescado y que una inversión que supera el millón de dólares puede ser recuperada en aproximadamente tres años de pesca al ritmo actual, todo esto sin considerar las ventas adicionales por el aceite de recuperación. Las perspectivas a mediano y largo plazo consisten en lograr una cada vez mayor eficiencia de tratamiento y recuperación del $A B$ así como en la implementación de este sistema en todas las fábricas harineras de TASA [5].

Además de TASA, diversas empresas pesqueras están invirtiendo en aplicar tecnología limpia para mejorar sus procesos y contribuir a las políticas de producción limpia. Casos como este son: la instalación del sistema de gas natural en la Planta Pisco de Pesquera Diamante, lo que convierte a Diamante en la primera empresa pesquera de la zona operando con energía más limpia y eficiente. La implementación de todo el sistema implicó una inversión significativa por parte de la empresa y la puesta en marcha de un programa de capacitación de todo el personal para el correcto uso de esta nueva fuente de energía [9].

Procesos similares están siendo replicados en las principales empresas del sector pesquero industrial peruano. Cada uno de ellos con sus propios sistemas de recuperación pero todos basados en los principios de prevención y conservación del medio ambiente marino.

Casos de ecoeficiencia empresarial en el sector pesquero están liderados por las empresas que vienen implementando medidas ecoeficientes con la finalidad de incentivar el aprovechamiento 
eficiente de los recursos naturales y promover la protección de la calidad ambiental. Contribuyendo así, a la competitividad del país y facilitando su transición hacia el desarrollo sostenible. En este sentido la empresa pesquera Austral Group S.A.A. fue premiada con el de premio a la ecoeficiencia en el 2010 otorgado por el Ministerio del Ambiente con el proyecto "Sistema de gestión ambiental" pues demostró la implementación de la ecoeficiencia de manera integral en toda su actividad empresarial la cual incluyó los rubros de: Ecodiseño, Buenas prácticas ambientales, Producción más limpia, Manejo sostenible de los recursos naturales renovables y la Gestión externa de residuos [8].

\section{CONCLUSIONES}

Las principales conclusiones de la investigación son las siguientes:

La Producción Más Limpia (PML) introduce una concepción básica para el mejoramiento del desempeño de las empresas. Mientras que instrumentos y mecanismos de comando y control tradicionales obligan a las empresas de cumplir con unos parámetros administrativos que a su vez pueden, pero no necesariamente, influir en ajustes en procesos productivos. Mientras que, la aplicación de PML por parte de las empresas, asegura cambios estructurales.

Siendo los efluentes el principal agente contaminante del medio marino, es necesario que las empresas productoras de harina y aceite de pescado inicien una migración hacia procesos de producción más limpia mediante la aplicación de tecnologías limpias que permitan cumplir con las normas legales, mejorar el desempeño ambiental, mejorar la eficiencia de sus procesos mediante la incorporación de sólidos recuperados en el proceso productivo principal así como mejorar su imagen ante la comunidad y facilitar el acceso a mercados internacionales.

Si bien la ecoeficiencia y producción más limpia se orientan han la prevención de la contaminación, la diferencia es que la primera la centra en la producción de bienes y servicios con menor consumo de insumos y materias primas y menor contaminación ambiental, mientras que la segunda implica un cambio en las prácticas culturales o procesos productivos a través de la inclusión de nuevas tecnologías conocidas como "tecnologías limpias" para lograr la reducción de la contaminación o mejorar el desempeño ambiental.

La aplicación de tecnologías limpias en el sector industrial entre ellos en la producción de harina y aceite de pescado ha venido llevándose paulatinamente con la adecuación de equipos e instalación de nuevas etapas del proceso productivo principal a fin de reducir el efecto contaminante de las emisiones gaseosas y material particulado, residuos sólidos y los efluentes en el medio ambiente.

Las diversas experiencias de las empresas del sector pesquero industrial, muestran que la toma de decisiones para adoptar alternativas preventivas es el resultado de una decisión gerencial la cual está íntimamente relacionada con la concepción de los directivos de la empresa sobre el valor agregado de la gestión ambiental para su negocio. La percepción de este valor depende de factores del entorno de la empresa como son presiones de mercados, presiones de autoridades, oferta de servicios o facilidades de financiación, reconocimiento, entre otros.

\section{REFERENCIAS BIBLIOGRÁFICAS}

[1] Arroyave, J., \& Garcés, L. (2007). Tecnologías ambientalmente sostenibles. http://repository.lasallista.edu.co/dspace/ bitstream/10567/513/1/pl_v1n2_78-86_ tecnolog\%C3\%ADas.pdf)

[2] Badillo, T. (2009). Pesca y producción limpia. factibilidad de una microempresa de extracción de camarón con tecnología de bajo impacto ambiental. Revista Desarrollo Gerencial. No. 1: 84-97. http://publicaciones.unisimonbolivar. edu.co/rdigital/desarrollogerencial/index.php/ desarrollogerencial/article/viewFile/12/10.

[3] Centro de Promoción de Tecnologías Sostenibles (CPTS), USAID/Bolivia \& Embajada Real De Dinamarca (2005). Guía Técnica General de Producción Más Limpia http://libroweb.alfaomega.com.mx/catalogo/ pmlproduccionmaslimpia/libreacceso/reflector/ ovas_statics/unid5/PDF_Espanol/Guia_ Tecnica_PML.pdf)

[4] Centro de Producción más Limpia - Consejo Nacional de Producción Limpia. (s. f.). Chile. Recuperado 17 de marzo de 2014, a partir de http://www.cpl.cl/Acuerdos(APL)/sector. php?id=25

[5] Centro para la Sostenibilidad Ambiental CSA (2007). Universidad Peruana Cayetano Heredia. http://www.anchoveta.info/index. php?option=com_content\&task=view\&id=32\&lt emid=91.) 
[6] Dalila, G. (2012). Sistema de producción más limpia para el manejo de Efluentes en el laboratorio de pruebas físicas de cuero de la asociación nacional de curtidores del Ecuador - Ance Producción Más Limpia. Proyecto de Trabajo de Graduación. Universidad Técnica de Ambato Facultad de Ingeniería en Sistemas Electrónica e Industrial http://repo.uta.edu. ec/bitstream/handle/123456789/2329/Tesis_ t686id. pdf?sequence=1.

[7] Estrucplan (2006). Guía de Producción Más Limpia - Perú - Parte 01. https://www. estrucplan.com.ar/Producciones/entrega. asp?IdEntrega=1389

[8] Ministerio del Ambiente (MINAM). Ecoeficiencia empresarial 2010, http://cdam.minam.gob.pe/ novedades/ecoeficienciaempresarial.pdf

[9] Pesquera Diamante (2014). Un año de logros para pesquera diamante. http://www.diamante. com.pe/wps/wcm/connect/Diamante_es/home/ opt/noticias/2014?not=1

[10]Plan estratégico nacional 2011-2015. Perú. http://www.peru.gob.pe/docs/PLANES/102/ PLAN_102_Plan_Estrat\%C3\%A9gico_
Sectorial_Multianual_\% 28 PES EM\%29_2011-2015_2011.pdf.

[11]Produce. (2008). Límites Máximos Permisibles (LMP) para la Industria de Harina y Aceite de Pescado y Normas Complementarias. Decreto Supremo 010-2008-PRODUCE.

[12]Retamoso, C. (2007). Producción Limpia, Contaminación y gestión ambiental. http:// books.google.es/books?hl=es\&lr=\&id=ea0kufq BmtQC\&oi=fnd\&pg=PA17\&dq=produccion + ma $s+l i m p i a \& o t s=x d L r r R 8 I g k \& s i g=g g C Q u d 341 j k V$ X6KK1wEJA3tHPWU\#v=onepage\&q=producci on $\% 20$ mas $\% 20$ limpia\&f=false)

[13]Rodríguez, J., Hleap, J., Estrada, F., Clavijo, J. \& Perea, N. (2011). Gestión de residuos pecuarios de sistemas de producción más limpia http://www.bdigital.unal.edu. co/11644/1/9789587610123.pdf.

[14]VanHoof,B., \&Herrera, C. (2007).Producciónmás limpia en Colombia http://libroweb.alfaomega. com.mx/catalogo/pmlproduccionmaslimpia/ libreacceso/libreacceso/reflector/ovas_statics/ unid1/PDF_Espanol/PML_Colombia.pdf. 